\title{
Assessment of disinfection of hospital surfaces using different monitoring methods ${ }^{1}$
}

\author{
Adriano Menis Ferreira ${ }^{2}$ \\ Denise de Andrade ${ }^{3}$ \\ Marcelo Alessandro Rigotti ${ }^{4}$ \\ Margarete Teresa Gottardo de Almeida ${ }^{5}$ \\ Odanir Garcia Guerra² \\ Aires Garcia dos Santos Junior ${ }^{6}$
}

Objective: to assess the efficiency of cleaning/disinfection of surfaces of an Intensive Care Unit. Method: descriptive-exploratory study with quantitative approach conducted over the course of four weeks. Visual inspection, bioluminescence adenosine triphosphate and microbiological indicators were used to indicate cleanliness/disinfection. Five surfaces (bed rails, bedside tables, infusion pumps, nurses' counter, and medical prescription table) were assessed before and after the use of rubbing alcohol at 70\% (w/v), totaling 160 samples for each method. Non-parametric tests were used considering statistically significant differences at $p<0.05$. Results: after the cleaning/disinfection process, $87.5,79.4$ and $87.5 \%$ of the surfaces were considered clean using the visual inspection, bioluminescence adenosine triphosphate and microbiological analyses, respectively. A statistically significant decrease was observed in the disapproval rates after the cleaning process considering the three assessment methods; the visual inspection was the least reliable. Conclusion: the cleaning/disinfection method was efficient in reducing microbial load and organic matter of surfaces, however, these findings require further study to clarify aspects related to the efficiency of friction, its frequency, and whether or not there is association with other inputs to achieve improved results of the cleaning/disinfection process.

Descriptors: Staphylococcus Aureus; Equipment Contamination; Disinfection/Methods; 2-Propanol; Adenosine Triphosphate.

1 Supported by Fundação de Apoio ao Desenvolvimento do Ensino, Ciência e Tecnologia do Estado de Mato Grosso do Sul (FUNDECT), Brazil, process \# 23/200.299/2009, and Conselho Nacional de Desenvolvimento Científico e Tecnológico (CNPq), Brazil, process \# 152708/2007-3.

2 PhD, Professor, Universidade Federal de Mato Grosso do Sul, Três Lagoas, MS, Brazil.

${ }^{3} \mathrm{PhD}$, Associate Professor, Escola de Enfermagem de Ribeirão Preto, Universidade de São Paulo, PAHO/WHO Collaborating Centre for Nursing Research Development, Ribeirão Preto, SP, Brazil.

${ }^{4}$ Doctoral student, Faculdade de Medicina de São José do Rio Preto, São José do Rio Preto, SP, Brazil. Assistante Professor, Universidade Federal de Mato Grosso do Sul, Três Lagoas, MS, Brazil.

5 PhD, Professor, Faculdade de Medicina de São José do Rio Preto, São José do Rio Preto, SP, Brazil.

${ }^{6}$ Master's student, Universidade Federal de Mato Grosso do Sul, Campo Grande, MS, Brazil. RN, Hospital Nossa Senhora Auxiliadora, Três Lagoas, MS, Brazil.

Corresponding Author: Adriano Menis Ferreira

Universidade Federal de Mato Grosso do Sul. Curso de Enfermagem

Av. Ranulpho Marques Leal, 3484

Distrito Industrial

CEP: 79620-080, Três Lagoas, MS, Brasil

E-mail: a.amr@ig.com.br
Copyright $\odot 2015$ Revista Latino-Americana de Enfermagem This is an Open Access article distributed under the terms of the Creative Commons Attribution Non-Commercial License (CC BY-NC).

This license lets others distribute, remix, tweak, and build upon your work non-commercially, and although their new works must also acknowledge you and be non-commercial, they don't have to license their derivative works on the same terms. 


\section{Introduction}

It is indisputable that environmental contamination involving important microorganisms - MethicillinResistant Staphylococcus Aureus (MRSA), VancomycinResistant Enterococus, Acinetobacter spp., and Clostridium difficile, among others - represents a risk to patients and professionals. In this sense, studies corroborate the finding that cleaning and/or disinfecting environmental surfaces reduces contamination, and consequently, contributes to reducing the occurrence of infection ${ }^{(1-2)}$. Units occupied by individuals colonized or infected with Multidrug-resistant (MDR) strains constitute a risk to newly admitted patients if proper cleaning and disinfection of the inanimate environment is not accomplished ${ }^{(1,3-7)}$.

Acknowledging the importance the environment plays in the transmission of microorganisms, the Centers for Disease Control and Prevention and the Health Care Infection Control Practices Advisory Committee recommend surfaces in proximity to patients, which are frequently touched, be properly cleaned and disinfected and that health care facilities ensure its professionals adhere to such procedures ${ }^{(7-8)}$.

In this sense, the efficiency of cleaning and disinfection processes of inanimate surfaces, denoted here as the cleaning/disinfection procedure, should be investigated as a scientific process with measurable results. It can include methods to monitor the efficiency of cleaning/disinfection processes, such as visual inspection, culture testing for microorganisms, and also to detect organic matter by verifying the presence of Adenosine triphosphate (ATP) using bioluminescence, methods that have been available for more than 30 years $^{(3,6,9-13)}$.

We should clarify that the cleaning/disinfection of the environment results in removing dirt, reducing microbial load and eliminating multi-resistant strains, obviously, the intent of which, considering its purpose and the way it is performed, is not to achieve an environment free of microorganisms. The situation, however, is of concern given the process' operational failures, especially in areas housing patients at a high risk of acquiring infections, such as Intensive Care Units $(\mathrm{ICU})^{(5)}$.

Given the previous discussion, this study's objective was to assess the efficiency of the cleaning/disinfection process of surfaces of an ICU using conventional methods of inspection, ATP presence and identification of Staphylococcus aureus/MRSA.

\section{Method}

This descriptive-exploratory study with a qualitative approach was conducted over the course of four weeks in the medical-surgical ICU of a general hospital linked to the Brazilian Unified Health System (SUS). Data were collected between October and November 2011 . There was $100 \%$ occupancy during the entire period of study.

A convenience sample was used and the surfaces were selected based on the frequency of contact with hands, people traffic and proximity to patients: bed rails, bedside tables, infusion pump, nurses' counter, and medical prescription table. These surfaces were made of either stainless steel, painted steel, formica or granite.

The cleaning/disinfection protocol established in the facility included directly rubbing surfaces with a $100 \%$ cotton cloth dampened with hydrated ethyl alcohol at $70 \%(w / v)$, rubbing three times for at least 15 seconds.

The cleaning and disinfection routine of the surfaces included in the study was performed once a day by the nursing staff at the beginning of the morning shift. Considering that the assessments were performed in the morning shift as well, the surfaces probably went approximately 12 hours without cleaning/disinfection.

A cloth composed of $80 \%$ rayon, $15 \%$ polypropylene and $5 \%$ polyester folded into four parts and embedded in hydrated ethyl alcohol at 70\% (w/v) was used to rub surface three times for at least 15 seconds. In order to dampen the cloths completely, the disinfectant was sprayed 20 times on each cloth. A different cloth was used in each patient's unit and another was used for the nursing counter and medical prescription table and was replaced if every fold was visibly soiled.

\section{Tests utilized}

Data were collected before and after applying alcohol at $70 \%(\mathrm{w} / \mathrm{v})$ on surfaces. Ten minutes elapsed before the second collection ${ }^{(12)}$. All tests were performed by two researchers from Monday through Friday and included visual assessment, presence of ATP and identification of Staphylococcus aureus/MRSA, respectively ${ }^{(6,12)}$.

Levels of ATP bioluminescence $\left(3 \mathrm{M}^{\mathrm{m}}\right.$ Clean-Trace $^{\mathrm{m}}$ ATP System) were used to assess the efficiency of the cleaning/disinfection process using alcohol on surfaces in a $100 \mathrm{~cm}^{2}$ area for the bedside table, nursing counter, 
and medical prescription table and the entire bed rail and infusion pump panel. This technology detects ATP based on organic residue (human secretions, excretions and blood, food and other organic material) including viable and non-viable microbial load (probably recently killed microorganisms). Bioluminescence utilizes light to measure organic matter and this measure can be used as an indicator of hygiene. The light is emitted in direct proportion to the quantity of ATP present and is measured in Relative Light Units (RLU): the higher the reading, the higher the level of ATP present and, consequently, the higher the organic load. Therefore, monitoring ATP is a simple and quantitative method to monitor cleaning(3).

Petrifilm $^{\mathrm{m}}$ dishes $\left(3 \mathrm{M}^{\mathrm{m}}\right.$, St Paul, MN, USA) Staph Express $3 \mathrm{M}^{\mathrm{T}}$ model, prepared with BairdParker modified chromogenic media, were used for presumptive detection of Staphylococcus aureus and MRSA. This medium is selective and differential for Staphylococcus aureus, with potential isolation confirmed by the DNase test. A sampling area of $30 \mathrm{~cm}^{2}$ and incubation at $37^{\circ} \mathrm{C}$, for $24-48 \mathrm{~h}$, was adopted for the Petrifilm ${ }^{\mathrm{Tm}}$ model.

Susceptibility to methicillin was verified by using a screening test for oxacillin resistance. Petri dishes containing Muller-Hinton agar supplemented with $4 \%$ $\mathrm{NaCL}$ and $6 \mu \mathrm{g}$ of oxacillin, known as MRSA medium (Probac do Brasil ${ }^{\circledR}$ ), were used. These microorganisms were sub-cultured in $\mathrm{BHI}$ broth and incubated at $37^{\circ} \mathrm{C}$ for 24 hours. After this period, they were inoculated on the dishes and incubated at $37^{\circ} \mathrm{C}$ for 24 and 48 hours. Any growth on the dish was considered to be MRSA.

The parameters described in Table 1 were used in the interpretation of the cleaning/disinfection process $^{(6,9,11)}$

In the conventional assessment, i.e., through visual inspection, surfaces were considered dirty if there was dust, waste (with or without organic matter), moisture or stains ${ }^{(11)}$.

In the statistical analysis, data were transferred to the SPSS (Statistical Package for Social Science), version 15.0. The Wilcoxon test was used for paired samples to check for ordinal variables (RLU and CFU), while the McNemar test with binomial distribution was used for the dichotomous variables (approved/ disapproved). Pearson's chi-square test or Fisher's exact test were used to compare the proportions of surfaces classified as clean. The level of significance adopted was $5 \%(p<0.05)$.
Table 1 - Monitoring of surface cleaning according to different methods. Três Lagoas, MS, Brazil, 2011

\begin{tabular}{lcc}
\hline \multicolumn{1}{c}{$\begin{array}{c}\text { Cleaning } \\
\text { assessment }\end{array}$} & Result & Interpretation \\
\hline Percentage of visually & $>70$ & Acceptable \\
clean surfaces & $60-69$ & Partially acceptable \\
& $<59$ & Unacceptable \\
ATP* bioluminescence & $<500 \mathrm{RLU} \dagger$ & Acceptable \\
& $>500 \mathrm{RLU}$ & Unacceptable \\
Staphylococcus aureus/ & $<1 \mathrm{ufc} \$ / \mathrm{cm}^{2}$ & Acceptable \\
MRSA & $>1 \mathrm{ufc} / \mathrm{cm}^{2}$ & Unacceptable \\
\hline
\end{tabular}

*Adenosine triphosphate

† Relative light units

₹ Methicillin-resistant Staphylococcus aureus

$\S$ Colony-forming units

\section{Results}

A total of 320 assessments were performed: 160 assessments (visual, ATP measurements, and Staphylococcus aureus/MRSA presence) were performed before the cleaning/disinfection process and 160 were performed after the process.

Before cleaning/disinfection, 90/160 (56.2\%) of the surfaces were considered clean, as there was no visible dirt. According to ATP measurement and verification of Staphylococcus aureus/MRSA, 44/160 (27.5\%) and $92 / 160(57.5 \%)$, respectively, were considered clean. Therefore, the cleaning rate of surfaces ranged from 27 to $57.5 \%$, depending on the assessment method. After the disinfection process, $140 / 160$ (87.5\%), 127/160 $(79.4 \%)$ and $140 / 160(87.5 \%)$ of the surfaces were considered clean using the ATP and microbiological methods, respectively $(p<0.05)$.

The percentage of surfaces that were not approved according to different methods varied considerably (Table 2).

Disapproval rates using the visual method after cleaning were statistically lower $(p<0.001)$, while the infusion pump was the only surface for which the difference was not statistically significant $(p=0.25)$. Note that the infusion pump was approved in more than $90 \%$ of cases even before alcohol was applied and there was no disapproval after cleaning. Differences in disapproval rates before and after rubbing alcohol when measuring ATP were statistically different $(p<0.001)$, ranging from 37.5 to $62.6 \%$. Similarly, the differences in disapproval rates from a microbiological perspective before and after cleaning/disinfection were statistically significant $(p<0.001)$, ranging from 12.5 to $46.8 \%$.

Differences in disapproval rates between visual assessment and ATP (Table 3) were statistically significant $(p<0.001)$ and ranged from 3.1 to $31.2 \%$. 
Differences in disapproval rates between the visual and microbiological methods (Staphylococcus aureus/MRSA) were not significant, with a range from 3.2 to $15.5 \%$. According to each surface, the differences between disapproval rates of cleaning/disinfection using ATP and microbiological methods are not significant and ranged from 0 to $28.1 \%$.
While disapproval rates provide an indicator of the efficiency of applying alcohol at $70 \%$ in regard to the standards determined by ATP readings, they do not indicate the extent to which cleanliness/disinfection was disapproved. Hence, ATP readings in RLU, which were obtained before and after the cleaning/disinfection process on the five surfaces, varied considerably (Table 4).

Table 2 - Disapproval rates before and after the cleaning/disinfection process using alcohol at $70 \%$ assessed through three methods. Três Lagoas, MS, Brazil, 2011

\begin{tabular}{|c|c|c|c|c|c|c|}
\hline \multirow{3}{*}{ Surfaces } & \multicolumn{6}{|c|}{$\%$ Disapproval } \\
\hline & \multicolumn{3}{|c|}{ Before cleaning } & \multicolumn{3}{|c|}{ After cleaning } \\
\hline & Visual & ATP* & $\begin{array}{c}\text { S. aureus/ } \\
\text { MRSA† }\end{array}$ & Visual & ATP & $\begin{array}{l}\text { S. aureus/ } \\
\text { MRSA }\end{array}$ \\
\hline Bed rail $(n=32)$ & 59.4 & 97 & 53.1 & 18.8 & 50 & 22 \\
\hline Bedside table $(n=32)$ & 81.3 & 84.4 & 56.2 & 37.5 & 22 & 22 \\
\hline Infusion pump (n=32) & 9.4 & 53.1 & 56.2 & 0 & 15.6 & 9.4 \\
\hline Nursing counter $(n=32)$ & 47 & 72 & 15.6 & 6.3 & 12.5 & 3.1 \\
\hline Prescription table $(n=32)$ & 22 & 59.4 & 31.2 & 0 & 3.1 & 6.2 \\
\hline Total $(n=160)$ & 43.7 & 72.5 & 42.5 & 12.5 & 20.6 & 12.5 \\
\hline
\end{tabular}

* Adenosine triphosphate

† Methicillin-resistant Staphylococcus aureus

Table 3 - Differences found in regard to the disapproval rates obtained with visual inspection of cleanliness/disinfection with alcohol at 70\% and ATP and microbiological methods. Três Lagoas, MS, Brazil, 2011

\begin{tabular}{|c|c|c|}
\hline \multirow{2}{*}{ Surfaces } & \multicolumn{2}{|c|}{ After cleaning } \\
\hline & ATP* (\%) & S. aureus/MRSA† (\%) \\
\hline Bed rail $(n=32)$ & 31.2 & 3.2 \\
\hline Bedside table $(n=32)$ & 15.5 & 15.5 \\
\hline Infusion pump (n=32) & 15.6 & 9.4 \\
\hline Nursing counter $(n=32)$ & 6.2 & 3.2 \\
\hline Prescription table $(n=32)$ & 3.1 & 6.2 \\
\hline
\end{tabular}

* Adenosine triphosphate

+ Methicillin-resistant Staphylococcus aureus

Table 4 - Adenosine triphosphate readings for the ICU's different surfaces before and after cleaning/disinfection with alcohol at 70\%. Três Lagoas, MS, Brazil, 2011

\begin{tabular}{|c|c|c|c|c|c|c|c|}
\hline \multirow[b]{2}{*}{ Surfaces } & \multicolumn{3}{|c|}{ Before cleaning } & \multicolumn{3}{|c|}{ After cleaning } & \multirow[b]{2}{*}{ pt } \\
\hline & $\begin{array}{c}\text { Average } \\
\text { (RLU)* }^{*}\end{array}$ & $\begin{array}{c}\text { Median } \\
\text { (RLU) }\end{array}$ & $\begin{array}{c}\text { Variation } \\
\text { (RLU) }\end{array}$ & $\begin{array}{c}\text { Average } \\
\text { (RLU) }\end{array}$ & $\begin{array}{c}\text { Median } \\
\text { (RLU) }\end{array}$ & $\begin{array}{c}\text { Variation } \\
\text { (RLU) }\end{array}$ & \\
\hline Bed rail $(n=32)$ & 21849.69 & 1999.5 & $185-576111$ & 1712.19 & 478.5 & $95-16799$ & $<0.001$ \\
\hline Bedside table $(n=32)$ & 2081.06 & 807 & $240-11303$ & 402.94 & 289.5 & $65-1777$ & $<0.001$ \\
\hline Infusion pump (n=32) & 692.03 & 523.5 & $105-3788$ & 249.38 & 139 & $34-1112$ & $<0.001$ \\
\hline Nursing counter $(n=32)$ & 1161.69 & 653 & $164-12154$ & 359.34 & 154.5 & $48-3305$ & $<0.001$ \\
\hline Prescription table $(n=32)$ & 1068.44 & 572 & $161-10309$ & 254.16 & 187 & $44-1112$ & $<0.001$ \\
\hline Total $(\mathrm{N}=320)$ & & & & & & & \\
\hline
\end{tabular}

*Relative light unit

+ Wilcoxon's test

The proportion of surfaces, the RLU's medians of which were lower after cleaning than before, were: 29 (90.6\%) out of 32 bed rails; 29 (90.6\%) out of 32 bedside tables; $28(87.5 \%)$ out of 32 infusion pumps; 30 (94\%) out of 32 nursing tables; and 29 (90.6\%) out of 32 prescription tables. RLU's medians obtained after cleaning/disinfection were lower than those obtained before $(p<0.001)$ for all the surfaces. Note that the bedrail was the surface out of all the surfaces inspected that presented the most dirt after cleaning/disinfection, with median $=478.5$. 
Counting of Staphylococcus aureus colonies was lower after cleaning for 21 (65.7\%) out of 32 bed rails; $23(71.9 \%)$ out of 32 bedside tables; 22 (69\%) out of 32 infusion pumps; 5 (15.7\%) out of 32 nursing tables; and 24 (75\%) out of 32 medical prescription tables. In general, there were significant statistical differences in reducing colony-forming units of Staphylococcus aureus for all the surfaces after cleaning/disinfection, with the exception of nursing tables $(p=0.072)$.

Methicillin-resistant Staphylococcus aureus was found before cleaning on 6 (19\%) out of 32 bed rails; $12(37.5 \%)$ out of 32 bedside tables; 9 (28\%) out of 32 infusion pumps; 2 (6.2\%) out of 32 nursing tables; and $6(19 \%)$ out of 32 prescription tables. Positive samples were found after cleaning on $4(12.5 \%)$ out of 32 bed rails; 4 (12.5\%) out of the 32 bedside tables; 3 (9.4\%) out of 32 infusion pumps; 2 (6.2\%) out of 32 nursing tables; and 1 ( $3 \%$ ) out of 32 medical prescription tables. Therefore, 35 (22\%) out of 160 microbiological samples tested positive for MRSA before cleaning/disinfection and $14(9 \%)$ out of 160 samples tested positive for MRSA $(p<0.05)$ after rubbing alcohol.

\section{Discussion}

Research(2-3,6,13) shows that the cleaning of patients' units is often deficient and surfaces can remain contaminated after the process. In this facility and in other hospital facilities, visual inspection has been often adopted as the single criterion to assess this process. Note, however, that surfaces that meet the visually-clean criterion may remain contaminated by microorganisms or other organic matter(2-3,11-12,14-15).

This study revealed that, based on visual inspection, $56.2 \%$ of the surfaces were classified clean before rubbing alcohol was used, thus, with unacceptable levels of cleanliness ${ }^{(11)}$. This situation changed after alcohol was rubbed on the surfaces, reaching acceptable levels of $87.5 \%$. In this sense, after use of rubbing alcohol at $70 \%(\mathrm{w} / \mathrm{v})$, the surfaces' levels of contamination were significantly reduced, considering that similar results were found among the different assessment methods. A possible explanation for ATP assessment having reached results close to the visual assessment after cleaning (79.4 and $87.5 \%$ ) is alcohol's efficacy in removing $\operatorname{dirt}^{(16)}$.

It is known that cleaning has distinct objectives, one of which is to improve or restore an environment's appearance, maintain its function and prevent deterioration. Considering microbiological contamination, cleaning reduces the number of microorganisms and any substance that may serve as a substrate for its growth or which may interfere in the subsequent processes of disinfection or sterilization $^{(14-15)}$. Hence, the term cleaning may be interpreted differently based on its purpose(11). The terms cleaning/disinfection were used here because a sanitizer (detergent, disinfectant or alcohol-based solution) was used, which has been demonstrated in a recent study ${ }^{(16)}$ to have, in addition to its antimicrobial action, a cleaning property that is visually assessed, a fact not previously considered.

Note that the classic and consensual recommendation of safe methods for the disinfection of surfaces consists of first cleaning the surface and then disinfecting it with a microbicidal agent ${ }^{(7-8)}$. In this study, however, the cleaning stage involving water and soap/detergent was not performed, because it is not a practice used in the facility under study. In fact, the direct application of alcohol on surfaces without prior cleaning is relatively frequent in healthcare facilities ${ }^{(16)}$. A recent study ${ }^{(16)}$ shows that the disinfecting efficacy of rubbing alcohol at $70 \%$ $(w / v)$ remains the same regardless of contaminated surfaces having being previously cleaned or not.

Note that the rate of approval using the visual method before cleaning was $56.2 \%$, compared to $27.5 \%$ approval when using the ATP method. This means that $28.7 \%$ of the surfaces were considered clean when they were actually dirty; i.e., organic matter was present (ATP).

A total of $87.5,79.4$ and $87.5 \%$ of the surfaces assessed by visual inspection, adenosine triphosphate bioluminescence and microbiological analyses, respectively, were considered clean after the cleaning/ disinfection process.

Another study(11) reports that $90 \%$ of the surfaces were considered clean according to visual inspection after the cleaning process, but only $10 \%$ of the surfaces resulted in $<2.5$ colony-forming units $/ \mathrm{cm}^{2}$, according to the microbiological analysis. Another study(10) reports that $93.3 \%$ of the surfaces were visually clean, $92 \%$ were microbiologically clean and $71.5 \%$ were free of organic matter. A more recent study ${ }^{(2)}$ was conducted in an ICU over the course of 14 days to describe the cleaning/disinfection conditions of four near-to-patient surfaces after the cleaning process and verified that 20,80 and $16 \%$ of the surfaces 
were disapproved when using the visual method, ATP and Staphylococcus aureus/MSRA analyses, respectively. There were statistically significant differences $(p<0.05)$ in cleanliness disapproval rates when comparing the ATP analysis with the visual and microbiological methods. The differences found in this study between the disapproval rates obtained through visual inspection and the ATP method (Table 3) were statistically significant ( $p<0.001)$; however, differences found between the visual and microbiological analyses and between ATP and microbiological analysis were not significant.

It is clear that quantitative methods are desirable to assess appropriately the efficiency of the cleaning/ disinfection process of surfaces in hospital and extrahospital environments(2-3,6,9-10,12). There is, though, a lack of indicators of what would be ideal results to obtain after the procedure. The cut-off points used in this study for classifying surfaces as clean have been proposed by authors ${ }^{(2,6,9,10-12)}$ to be appropriate, but no prospective studies have associated a reduction in the transmission of microorganisms and hospital-acquired infections with these parameters.

Therefore, using visual inspection as the sole criterion to assess cleanliness is not recommended since, in addition to the fact that subjectivity interferes in the process, there is a risk that an apparently clean area hides substrates and/or microbial contamination. In summary, the visual inspection method used in this study, as demonstrated by others, is the least sensitive method to assess cleanliness when compared to the bioluminescence ATP method(6,10-12,15).

Only recently, the Brazilian Health Surveillance Agency (ANVISA) published a manual(17) addressing the cleaning and disinfection of surfaces, a fact that shows a great advancement in the field. Unfortunately, however, it does not in detail describe more modern methods to assess cleanliness/disinfection of these surfaces, which may over-value the visual inspection as the easiest and most feasible method to be used.

It is expected that, in addition to removing microorganisms from a surface, alcohol will also reduce organic matter ${ }^{(16)}$ and this study shows that cleaning/disinfection using alcohol at $70 \%(\mathrm{w} / \mathrm{v})$ reduced organic matter measured by the ATP method for $79.4 \%$ of the surfaces.

Various studies indicated that the ATP monitoring is an important tool to inspect levels of cleanliness ${ }^{(3,10,12,15)}$. In this sense, as previously described, ATP analysis measures microbiological and non-microbiological sources, which can be removed by an effective cleaning/disinfection protocol. The test can be used to provide instantaneous feedback on the cleanliness of surfaces, working as an instrument to show deficiencies in cleaning/disinfection routines or techniques, and to assess protocols for and the training of the cleaning personnel(10,15). Additionally, as opposed to the visual test, the ATP method is not subjective and also has an advantage over the microbiological methods that require from 24 to 48 hours to provide results.

In regard to the presence of MRSA, it is important to note that from 1 to $27 \%$ of the surfaces of hospitals general units present this microorganism(4). The presence of MRSA before rubbing with alcohol was verified on $22 \%$ of surfaces and it still remained on $9 \%$ of the surfaces after cleaning despite 13\% drop in its incidence rate $(p<0.05)$. An investigation ${ }^{(12)}$ verified, through culture testing, that 40 (40\%) out of 100 samples tested positive for MRSA before cleaning and 24 (24\%) after cleaning, even though the sanitizer used was quaternary ammonium-based. Note that it is desirable that microorganisms are completely absent after cleaning/disinfection( ${ }^{(9)}$. Nonetheless, at this point, there is no evidence showing that the cleaning and/or disinfection protocols currently used for surfaces can completely eliminate multi-resistant strains. There is another consideration in regard to the cleaning and disinfection routine performed in the unit: the cleaning process was performed only once a day. Hence, the question is: would more frequent cleanings show more promising results?

Researchers $(6,10,12,18)$ monitoring the disinfection of surfaces using bioluminescence adenosine triphosphate and aerobic cultures show that cleaning and disinfection protocols are often disregarded. Another study(19) verified that $27 \%$ of the rooms remained contaminated with Acinetobacter baumannii and MRSA after four cycles of disinfection with sodium hypochlorite. A series of actions have to be involved in the cleaning/disinfection process for it consistently to eliminate microbial contamination, though time of contact with the disinfectant agent and intense friction are often valued.

The risk of acquiring MRSA was examined by researchers ${ }^{(20)}$ and a relationship between the hands of health workers and the area occupied by an infected or colonized patient was found. A total of $45 \%$ of 50 
healthcare workers acquired MRSA on their gloved hands through direct contact with patients and $40 \%$ of these same 50 professionals acquired MSRA from direct contact with surfaces.

It is worth noting that the hands of healthcare workers remain the main route of cross-infection transmission, if strict aseptic measures are not followed. Therefore, attention should be paid to highly contaminated sites that may compound the risk of infection even with appropriate adherence to hand hygiene ${ }^{(4,9,19)}$. It is estimated that from 20 to $40 \%$ of hospital-acquired infections have an etiology associated with cross-infection through the hands of health workers who become contaminated by direct contact with patients or indirectly by touching contaminated surfaces $^{(1,4,8-9,20)}$. It is no surprise that patients, healthcare workers, and visitors transfer secretions, oils, skin cells and organic matter to hospital surfaces. Through this physical contact, a film composed of inorganic salts, organic matter, and microorganisms accumulate over time and, presumably, facilitates the growth and transmission of viable microorganisms throughout the environment(21). Hence, the systematic implementation of cleaning and disinfection protocols for surfaces along with an assessment of their efficiency afterwards is justifiable(2-3,5,12,15).

In conclusion, as visual inspection alone does not provide reliable information about the risk of transmitting infections to patients, the surfaces in healthcare facilities should be assessed regarding the efficacy of cleaning processes. Fluorescent gel and visual assessment help to verify adherence to cleaning and disinfection protocols, while methods that monitor bio load (ATP and microbiological) provide a more relevant indication regarding the risk of infection and efficiency of the sanitizers used. If associated with a standardized monitoring regimen, ATP and/or microbiological analysis help to identify unacceptable levels of organic density and, consequently, the risk of surfaces acting as reservoirs of dirt and microorganisms, as long as such a regimen is systematically implemented and feedback is provided to workers. Additionally, such regimens should accurately interpret results to foresee clinical risks in a timely manner.

Therefore, the cleaning and disinfection routine with alcohol at $70 \%(\mathrm{w} / \mathrm{v})$ implemented more than once a day is desirable in the facility under study in order to achieve greater reduction of organic and microbial contamination. Corroborating this suggestion, the cleaning regimen using quaternary ammonium-based disinfectants have showed reduced bacterial load on bed rails by up to $99 \%$, though the microbial density, especially that of staphylococci, recovered rapidly: between 2.5 and 6.5 hours to reach the same levels prior to disinfection(21).

This study has some limitations, including the fact that the aerobic colonies on surfaces were not quantified, which would improve indicators of the quality of the cleaning and disinfection procedures. The study was performed in a single unit, which restricts generalization to other units in the same facility. There was a reduced number of samples for each surface due to limited financial resources and, finally, this study does not clarify the relationship of the presence of MSRA on surfaces with the risk of transmission to patients and healthcare workers.

\section{Conclusion}

The cleaning and disinfection process statistically $(p<0.001)$ reduced the disapproval rates according to the three assessment methods; visual inspection alone was not reliable in assessing the cleanliness/ disinfection levels of surfaces. In regard to MRSA, it was present on $22 \%$ of the surfaces before cleaning/ disinfection and reduced to $9 \%$ after the cleaning procedure $(p<0.05)$.

Further studies are required to determine objectively whether standardized cut-off values of the microbiological test and ATP analysis are accurate for the classification of surfaces in healthcare facilities as clean and also to clarify aspects related to the technique of friction or rubbing with a cloth, its frequency, and whether it is associated or not with other inputs such as disinfectants, especially in regard to the antimicrobial action on some multi-resistant microorganisms.

\section{Acknowledgment}

To Professor Vanderlei José Haas for providing support with the statistical analysis.

\section{References}


1. Otter JA, Yezli S, Salkeld JA, French GL. T. Evidence that contaminated surfaces contribute to the transmission of hospital pathogens and an overview of strategies to address contaminated surfaces in hospital settings. Am J Infect Control. 2013;41 Supp I5:6-11.

2. Ferreira AM, Andrade D de, Rigotti MA, Ferreira MVF. Condition of cleanliness of surfaces close to patients in an intensive care unit. Rev. Latino-Am. Enfermagem. 2011; 19(3):557-64.

3.Boyce JM, Havill NL, Havill HL, Mangione E, Dumigan DG, Moore BA. Comparison of fluorescent marker systems with 2 quantitative methods of assessing terminal cleaning practices. Infect Control Hosp Epidemiol. 2011;32(12):1187-93.

4. Dancer SJ. Importance of the environment in meticillin resistant Staphylococcus aureus acquisition: the case for hospital cleaning. Lancet Infect Dis. 2008;8:101-13.

5. Goodman ER, Platt R, Bass R, Onderdon AB, Yokoe DS, Huang SS. Impact of an environmental cleaning intervention on the presence of methicillin-resistant Staphylococcus aureus and vancomycin-resistant enterococci on surfaces in intenstive care unit rooms. Infect Control Hosp Epidemiol. 2008; 29:593-9.

6. Mulvey D, Redding $P$, Robertson C, Woodall C, Kingsmore $P$, Bedwell $D$, et al. Finding a benchmark for monitoring hospital cleanliness. J Hosp Infect. $2011 ; 77(1): 25-30$

7. CDC-Center of Diseases Control and Prevention (USA). Guideline for Environmental Infection Control in Health- Care Facilities: Recommendations of CDC and the Healthcare Infection Control Practices Advisory Committee (HICPAC). MMWR. [Internet]. 2003; [acesso 2 set 2013]; 52(RR-10):1-48. Disponível em: http:// www.cdc.gov/ncidod/hip/enviro/guide.htm.

8. Siegel JD, Rhinehart E, Jackson M, Chiarello L. Health Care Infection Control Practices Advisory Committee. 2007 Guideline for isolation precautions: preventing transmission of infectious agents in health care settings. Am J Infect Control. 2007;35 Suppl 2:65-164.

9. Dancer SJ: How do we assess hospital cleaning? A proposal for microbiological standards for surface hygiene in hospitals. J Hosp Infect. 2004;56:10-5.

10. Sherlock O, O'Connell N, Creamer E, Humphreys $\mathrm{H}$. Is it really clean? An evaluation of the efficacy of four methods for determining hospital cleanliness. J Hosp Infect. 2009; 72:140-6.
11. Malik RE, Cooper RA, Griffith CJ. Use of audit tools to evaluate the efficacy of cleaning systems in hospitals. Am J Infect Control. 2003; 31:181-7.

12. Boyce JM, Havill NL, Dumigan DG, Golebiewski M, Balogun O, Rizvani R. Monitoring the effectiveness of hospital cleaning practices by use of an adenosine triphosphate bioluminescence assay. Infect Control Hosp Epidemiol. 2009;30:678-84.

13. Al-Hamad A, Maxwell S. How clean is clean? Proposed methods for hospital cleaning assessment. J Hosp Infect. 2008; 1:1-7.

14. Collins BJ. The hospital environment: how clean should a hospital be? J Hosp Infect. 1988;11 Suppl A:53-6.

15. Lewis T, Griffith C, Gallo M, Weinbren M. A modified ATP benchmark for evaluating the cleaning of some hospital environmental surfaces. J Hosp Infect. 2008;69:156-63.

16. Graziano MU, Graziano KU, Pinto FMG, Bruna CQM, Queiroz RQ. Lascala CA. Effectiveness of disinfection with alcohol $70 \%(w / v)$ of contaminated surfaces not previously cleaned. Rev. Latino-Am. Enfermagem. 2013;21(2):618-23.

17. Agência Nacional de Vigilância Sanitária (BR). Segurança do paciente em serviços de saúde: limpeza e desinfecção de superfícies. Agência Nacional de Vigilância Sanitária. Brasília: Anvisa; 2012. 118 p.

18. Havill NL, Havill HL, Mangione E, Dumigan DG, Boyce JM. Cleanliness of portable medical equipment disinfected by nursing staff. Am J Infect Control. 2011;39(7):602-4.

19. Manian FA, Griesenauer S, Senkel D, Setzer JM, Doll SA, Perry AM, et al. Isolation of Acinetobacter baumannii complex and methicillin-resistant Staphylococcus aureus from hospital rooms following terminal cleaning and disinfection: can we do better? Infect Control Hosp Epidemiol. 2011;32:667-72.

20. Stiefel U, Cadnum JL, Eckstein BC, Guerrero DM, Tima MA, Donskey CJ. Contamination of hands with methicillin-resistant Staphylococcus aureus after contact with environmental surfaces and after contact with the skin of colonized patients. Infect Control Hosp Epidemiol. 2011;32:185-7.

21. Attaway H.H 3rd, Fairey S, Steed LL, Salgado CD, Michels HT, Schmidt MG. Intrinsic bacterial burden associated with intensive care unit hospital beds: effects of disinfection on population recovery and 
mitigation of potential infection risk. Am J Infect

Control. 2012;40(10):907-12. 